\title{
Frequency-Tunable and Pattern Diversity Antennas for Cognitive Radio Applications
}

\author{
A. H. Ramadan, ${ }^{1}$ J. Costantine, ${ }^{1}$ Y. Tawk, ${ }^{2}$ C. G. Christodoulou, ${ }^{3}$ and K. Y. Kabalan ${ }^{1}$ \\ ${ }^{1}$ The ECE Department, American University of Beirut, Beirut 1107 2020, Lebanon \\ ${ }^{2}$ The ECCE Department, Notre Dame University-Louaize, P.O. Box 72, Zouk Michael, Lebanon \\ ${ }^{3}$ The Configurable Space Microsystems Innovations \& Applications Center (COSMIAC), University of New Mexico, Albuquerque, \\ NM 87131-0001, USA
}

Correspondence should be addressed to A. H. Ramadan; ramadan@ieee.org

Received 5 December 2013; Accepted 23 January 2014; Published 6 March 2014

Academic Editor: Mario Bkassiny

Copyright (C) 2014 A. H. Ramadan et al. This is an open access article distributed under the Creative Commons Attribution License, which permits unrestricted use, distribution, and reproduction in any medium, provided the original work is properly cited.

Frequency-tunable microstrip antennas, for cognitive radio applications, are proposed herein. The approach is based on tuning the operating frequency of a bandpass filter that is incorporated into a wideband antenna. The integration of an open loop resonator(OLR-) based adjustable bandpass filter into a wideband antenna to transform it into a tunable filter-antenna is presented. The same technique is employed to design a cognitive radio pattern diversity tunable filter-antenna. A good agreement between the simulated and measured results for the fabricated prototypes is obtained. The radiation characteristics of each designed tunable filter-antenna are included herein.

\section{Introduction}

The broad frequency allocation with the variety of the existing standards calls for reconfigurable and frequency agile microwave circuits to pave the way toward reconfigurable radio front-end architectures [1]. The "spectrum overcrowding" arising issue, which is due to the coexisting telecommunication standards in overlapping frequency bands, calls for flexible receiver architectures to efficiently share wireless resources [2]. Cognitive radios are expected to sense the occupancy or target any available channel in the entire spectrum and tolerate interferers at any frequency as well. These requirements put constraints on the antenna design to efficiently sense the frequency spectrum [3].

A wideband front-end may not necessarily be the optimum solution for software-defined/cognitive radio as it leads to a compromise in the transceiver performance, caused by limitations in the front-end components. Therefore, techniques of introducing flexible frequency discrimination, which include tunable bandpass, tunable bandstop filters, and tunable narrowband antennas, help reduce spurious spectral content in the transmitter and limit out-of-band interference in the receiver [4]. Besides the inherent gainbandwidth product limitations and fluctuations of wideband antennas, their outputs can be heavily corrupted by wideband noise and thus result in a low signal-to-noise (SNR) ratio. Wideband antennas require high-speed ADCs, which are power intensive and have higher quantization errors. Finally, the design for wideband operation leads to compromise in the transceiver performance, caused by limitations in the RF front-end components such as amplifiers, oscillators, and mixers. All the above issues bring in narrowband frequencytunable antennas, or tunable filter-antennas, as good candidates for use to sense the frequency spectrum. This is because tunable bandpass filtering provides flexible frequency discrimination, wideband suppression of unwanted interference, gain flatness over the operating frequency band, less disruption of the antenna's radiation characteristics, better processing of down-converted signals, ease of implementation, and good performance. Hence, the new cognitive radio 
antenna, for overlay spectrum operation, may be a singleport antenna system, which can be appropriately controlled to efficiently sense the frequency spectrum.

In [5], the concept of multidimensional spectrum sensing is introduced. Herein, the spectrum sensing term involves obtaining spectrum usage characteristics across multiple dimensions such as time, space, frequency, and code. It is not simply based on measuring the spectral content or measuring the radio frequency energy over the spectrum, as traditionally understood. The conventional definition of the spectrum opportunity, which is often defined as a band of frequencies that are not being used by the primary user of that band at a particular time in a particular geographic area, only exploits the frequency, time, and space dimensions of the spectrum. However, other dimensions, such as location, angle of arrival, and code need to be explored. For the angle of arrival dimension, secondary users can alter their transmission direction without creating any interference if they know the location or direction of primary users. The potential of dynamical beam-forming and simultaneous frequency/polarization reconfiguration of reflectarray structures for use in cognitive radio application to achieve multiplexing in frequency, angular, and polarization domains is reported in [6]. Therefore, frequency tuning is one dimension to exploit in cognitive radio antennas. However, tunable filter-antennas with a pattern diversity attribute add on the efficiency of spectrum sensing in cognitive radio networks. For instance, pattern diversity tunable filter-antennas help combat multipath fading and cochannel interference, while sensing the frequency spectrum.

In this paper, tunable filter-antennas for overlay cognitive radio applications are presented. A design approach, which is based on converting the wideband operation of an antenna into a tunable narrowband one via preselect filtering is discussed. A varactor-tuned bandpass filter is integrated into a wideband antenna to achieve frequency tunability without disturbing the radiation pattern. The SMV1405 varactor is utilized in this work [7]. The same technique is employed to design a pattern diversity tunable filter-antenna for cognitive radio applications. The paper is organized as follows. Section 2 discusses the integration of an OLR-based bandpass filter, with a tuning capability, into a wideband antenna. The proposed tunable filter-antenna for cognitive radio applications is presented in Section 3. Section 4 discusses the design of a cognitive radio pattern diversity tunable filter-antenna. Conclusions are presented in Section 5.

\section{Integration of an OLR-Based Bandpass Filter into a Wideband Antenna}

According to [8], a microstrip transmission line loaded with rectangular-shaped half-wavelength open loop resonators results in an overall structure that behaves as an effective medium with negative-valued permeability. Therefore, a bandstop operation will appear in the vicinity of the OLR's resonant frequency. This bandstop behavior is because of the nonsimultaneous change in the sign of the permeability $(\mu)$ and permittivity $(\varepsilon)$ of the medium. This nonparallel

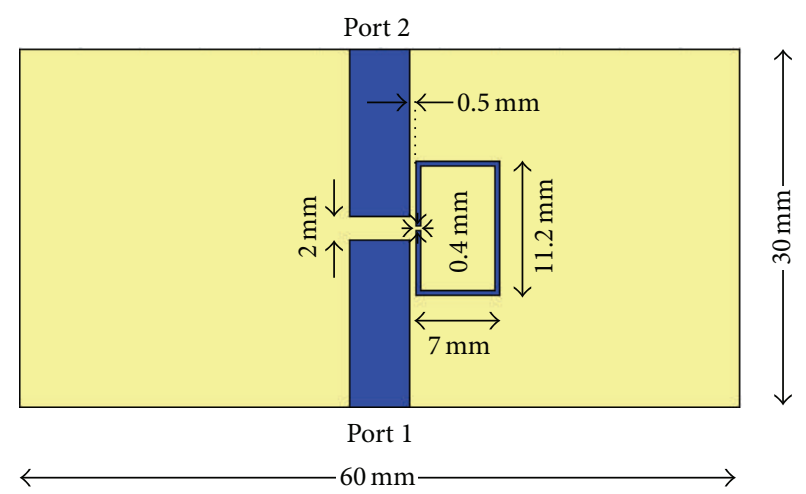

Figure 1: An OLR-based bandpass filter.

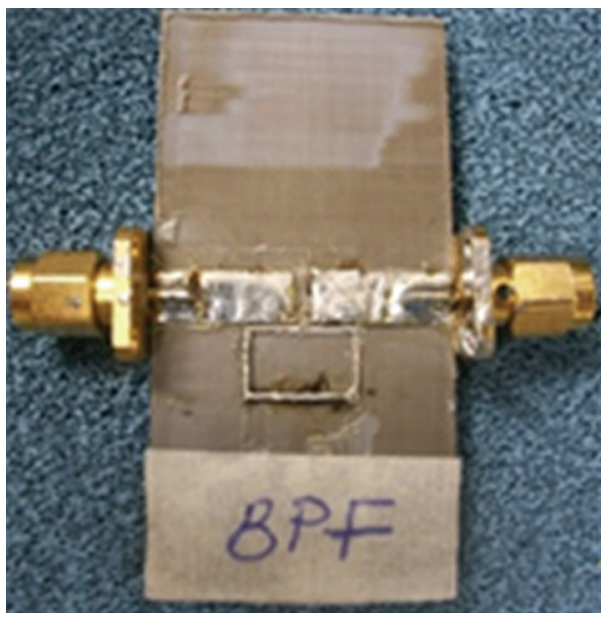

Figure 2: A photo of the fabricated bandpass filter prototype.

change affects the squared refractive index $\left(\eta^{2}\right)$ and yields no wave propagation. A bandpass transformation of the above scenario is obtained by etching a series capacitive gap in the microstrip transmission line, as shown in Figure 1. This combination is considered as a right-handed OLR/gap section and provides forward signal propagation in the frequency band where both the permeability and the permittivity are positive. The series capacitance loaded in the line precludes transmission outside of the OLR's resonant frequency band.

The illustrated OLR-based bandpass filter, which is based on a $1.6 \mathrm{~mm}$ thick Taconic TLY substrate, is designed and simulated using Ansoft HFSS [9]. A fabricated prototype of the filter is given in Figure 2. A comparison between the simulated and measured $S$-parameters is depicted in Figure 3, where a good agreement is attained. The integration of an OLR-based bandpass filter, into a wideband antenna, is discussed herein. A 3.41 GHz-operable OLR-based bandpass filter is firstly designed on a $1.6 \mathrm{~mm}$ thick Taconic TLY substrate. The dimensions of the proposed filter are given in Figure 4(a). A $60 \times 60 \times 1.6 \mathrm{~mm}^{3}$ Taconic TLY based wideband antenna is then designed as shown in Figure 4(b).

The antenna has a $30 \mathrm{~mm}$ long partial ground plane flushed with the feed line. A tapered matching section is incorporated between the rectangular patch of the antenna 


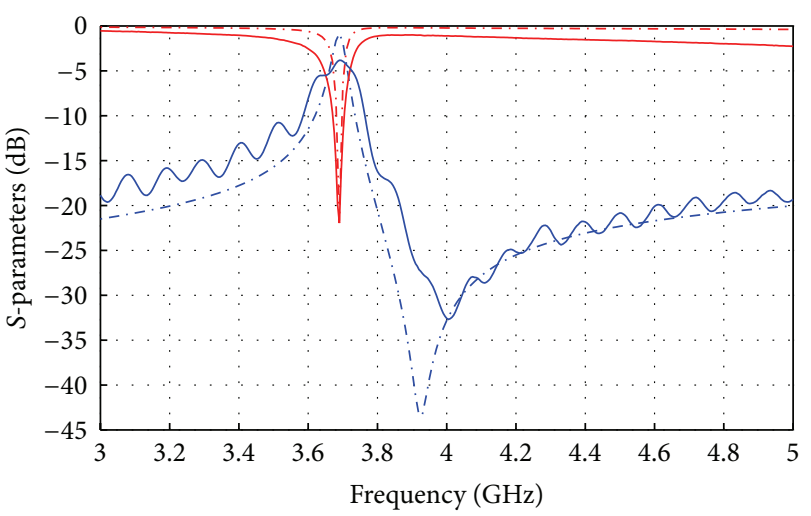

$$
\begin{array}{ll}
\text {-.- Simulated } S_{11} & - \text { Measured } S_{11} \\
\text {-.- Simulated } S_{21} & - \text { Measured } S_{21}
\end{array}
$$

Figure 3: Simulated and measured $S$-parameters of the OLR-based bandpass filter.

Port 2

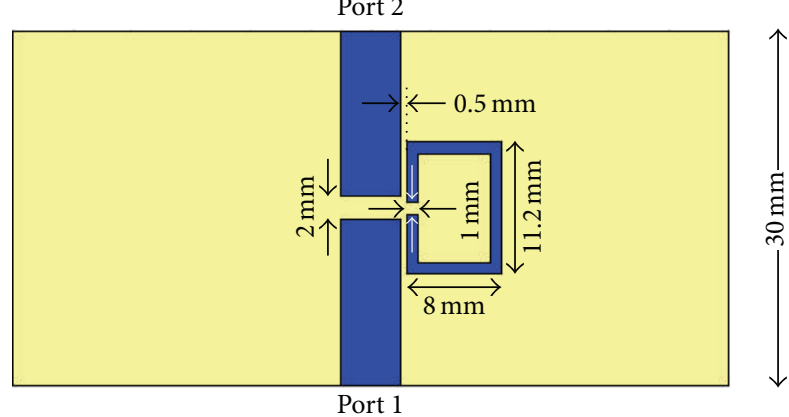

(a)

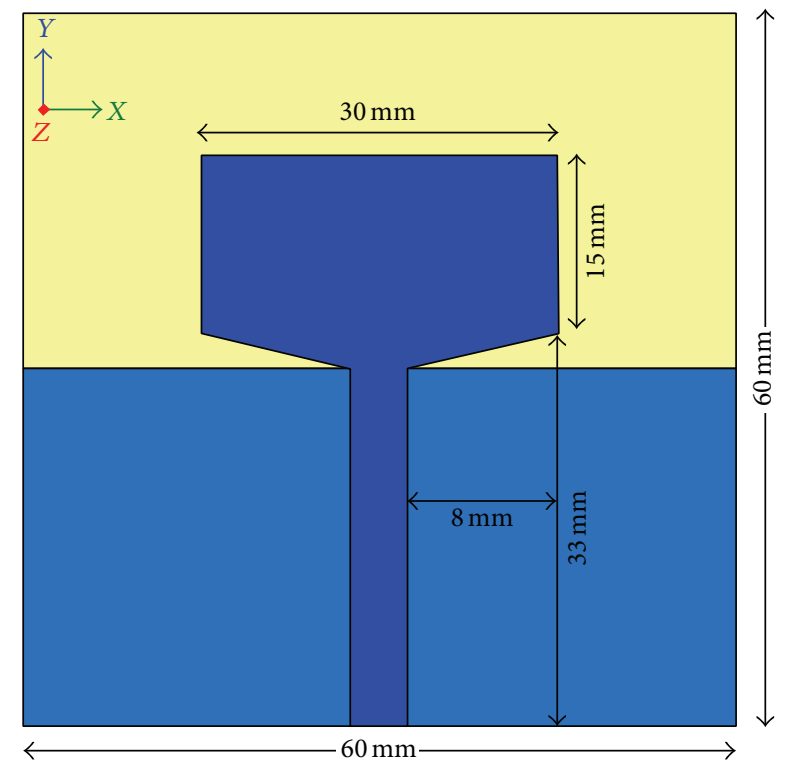

(b)

Figure 4: Geometry of (a) the OLR-based bandpass filter and (b) wideband antenna.

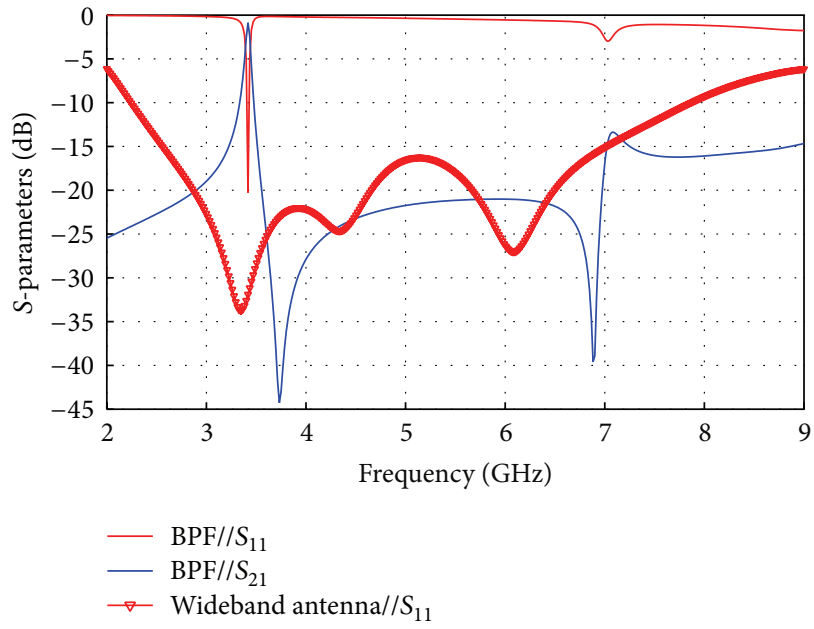

FIgURE 5: Computed $S$-parameters of the OLR-based bandpass filter and the wideband antenna.

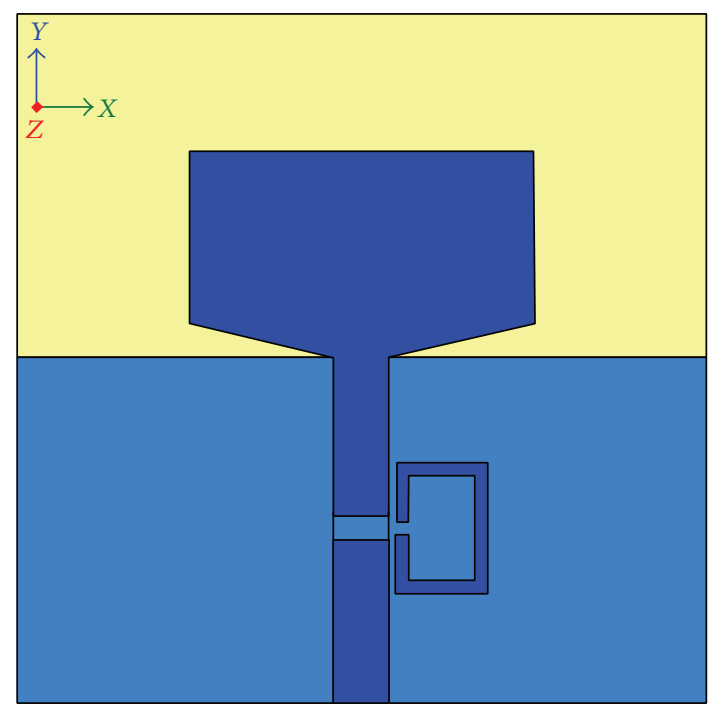

FIGURE 6: Integration of the OLR-based bandpass filter into the wideband antenna.

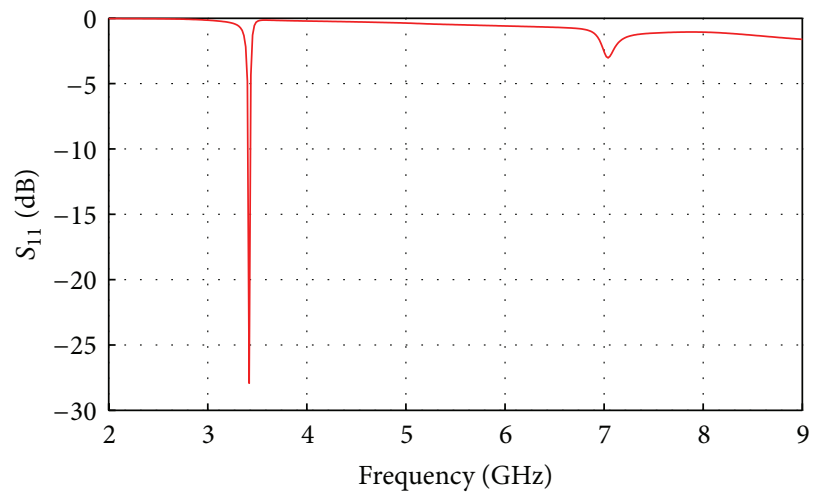

FIGURE 7: Simulated reflection coefficient of the assembled structure. 


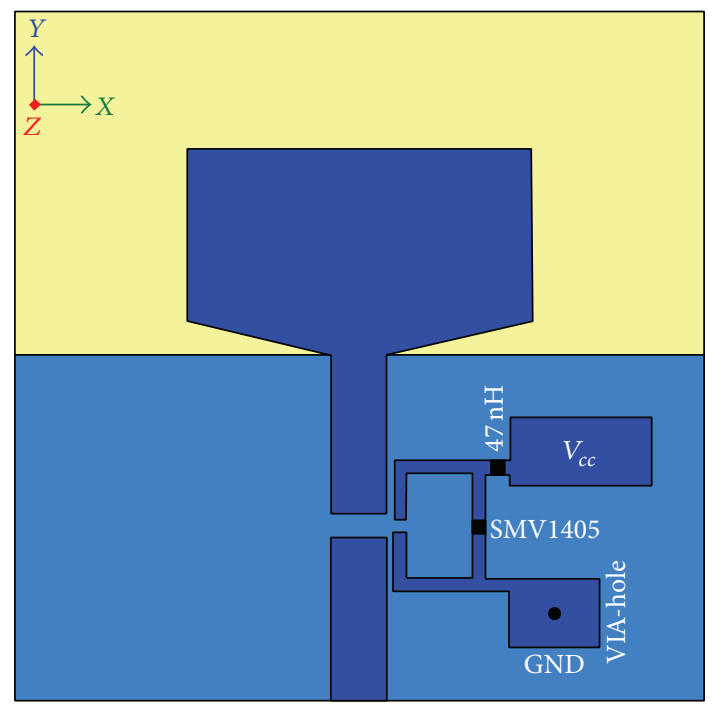

FIGURE 8: Configuration of the proposed tunable filter-antenna.

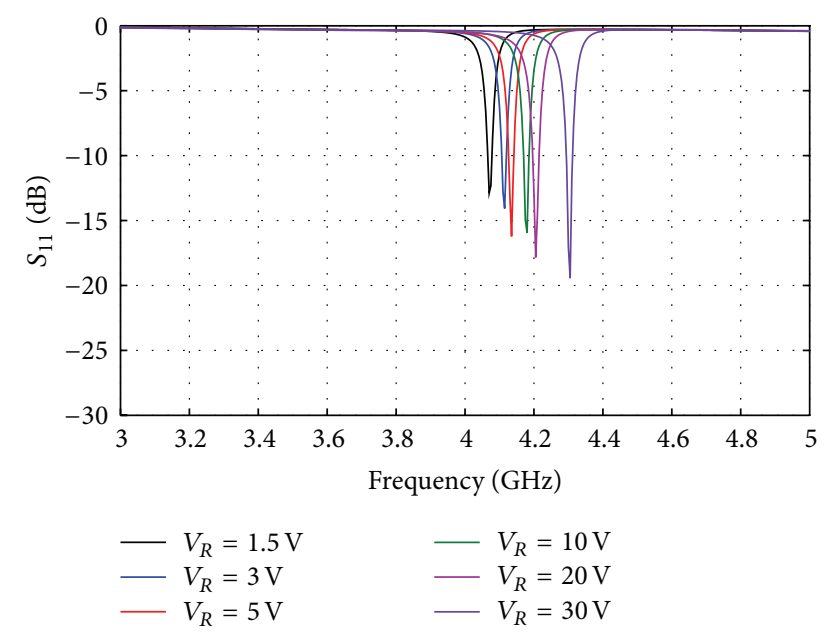

FIgURE 9: Simulated reflection coefficient of the proposed tunable filter-antenna.

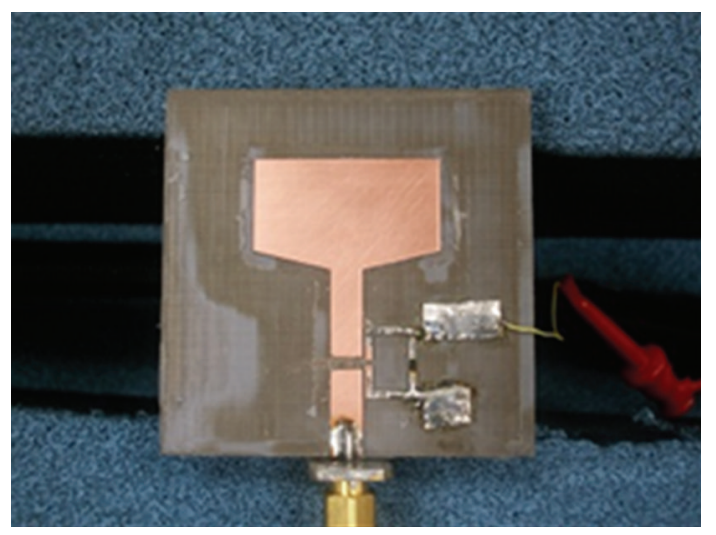

FIgURE 10: A fabricated prototype of the proposed tunable filterantenna.

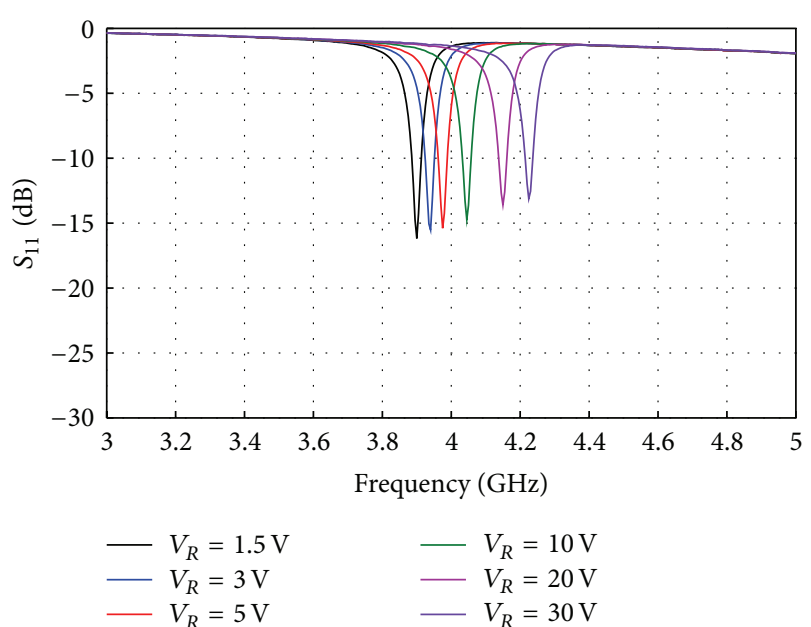

FIGURE 11: Measured reflection coefficient of the proposed tunable filter-antenna.

and its feed line in order to achieve better impedance matching. The computed $S$-parameters of the bandpass filter and the wideband antenna, in the $2-9 \mathrm{GHz}$ frequency range, are shown in Figure 5. The reflection coefficient of the antenna is $\leq-10 \mathrm{~dB}$ in the $2.24-7.86 \mathrm{GHz}$ frequency range. Finally, both the OLR-based bandpass filter and the wideband antenna are assembled in a single structure, as illustrated in Figure 6. The proposed filter-antenna is found to resonate, as shown in Figure 7, at the same operating frequency of the contained bandpass filter.

\section{A Narrowband Frequency-Tunable Antenna for Cognitive Radio Applications}

In order to achieve narrowband frequency tuning for the proposed $1.6 \mathrm{~mm}$ thick Taconic based filter-antenna, given that its radiation characteristics are not distracted, an SMV1405 varactor is mounted on the OLR contained in the bandpass filter to adjust its resonant frequency. According to [10], varactor-loaded half-wavelength OLRs witness longer electrical lengths and thus resonate at lower frequencies, as the loading capacitance is increased. In other words, the adjustment of the reverse voltage across the bridging varactor will result in tuning the resonant frequency of the filterantenna. The configuration of the proposed tunable filterantenna, along with its biasing network, is given in Figure 8. A $47 \mathrm{nH}$ RF choke is incorporated to prevent any RF leakage to the DC supply. The other terminal of the tunable OLR is grounded through a via-hole. Accordingly, the Vcc and GND pads are used to adjust the reverse voltage across the mounted varactor.

The computed reflection coefficient for different reverse voltages is shown in Figure 9. It is worth mentioning that as the reverse voltage increases, the tunable filter-antenna resonates at higher frequencies. This is because the value of the loading capacitance is inversely proportional to the applied reverse voltage, and this yields a resonance at higher frequencies. A prototype of the designed tunable 


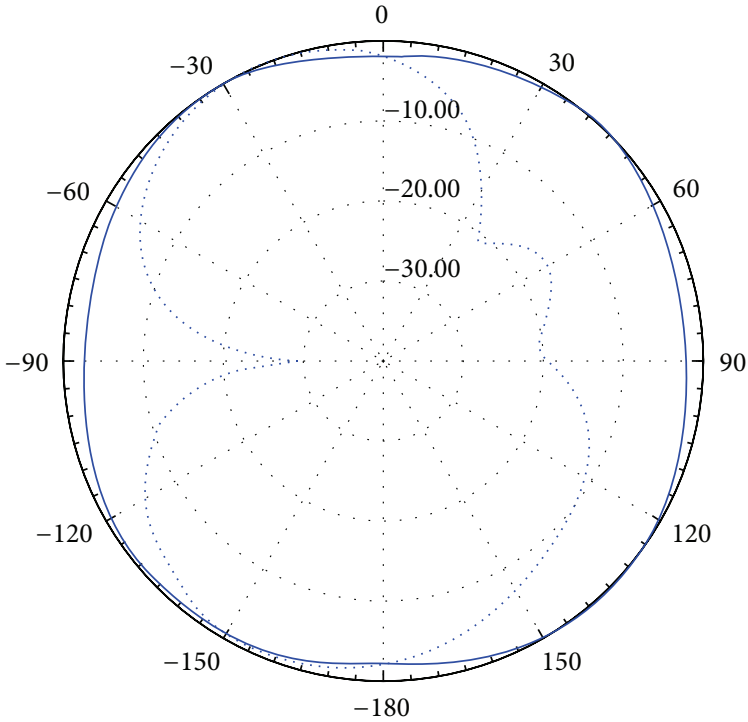

(a)

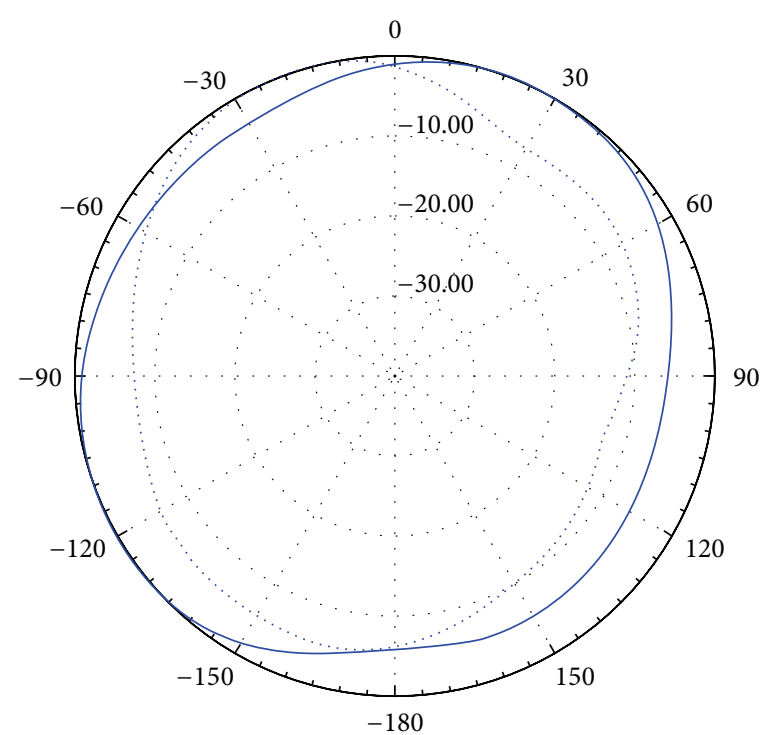

(b)

FIGURE 12: Normalized radiation pattern of (a) the wideband antenna and (b) the proposed tunable filter-antenna at 4.18 GHz (X-Z/Y-Z plane: solid/dotted line).

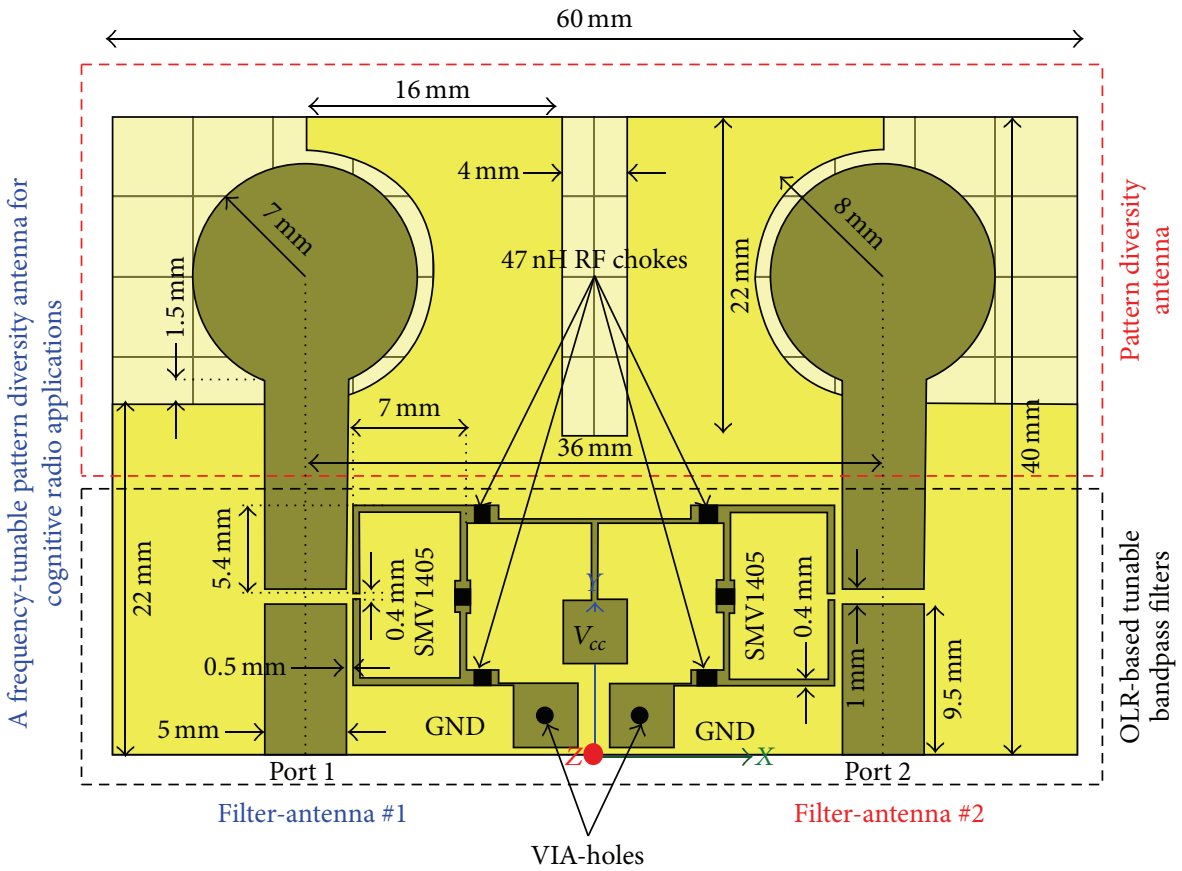

FIGURE 13: Configuration of the proposed pattern diversity tunable filter-antenna.

filter-antenna, as illustrated in Figure 10, is fabricated and measured. A good analogy between simulated and measured plots, as given in Figure 11, is revealed. However, a slight shift is witnessed due to Ansoft-HFSS varactor modeling issues and milling effects. The normalized radiation patterns, of the wideband antenna and the proposed tunable filter-antenna, at $4.18 \mathrm{GHz}$ are depicted in Figure 12. It is pretty obvious that the radiation pattern of the tunable filter-antenna, in the X$\mathrm{Z}$ plane, is still omnidirectional and minimally distracted.
Moreover, the gain of the tunable filter-antenna at $4.18 \mathrm{GHz}$ is $4 \mathrm{~dB}$, which is $1.86 \mathrm{~dB}$ higher than that of the wideband antenna at the same frequency.

\section{A Frequency-Tunable Pattern Diversity Antenna for Cognitive Radio Applications}

In a communication system implementing diversity, the same data is sent over independent fading paths. The signals 


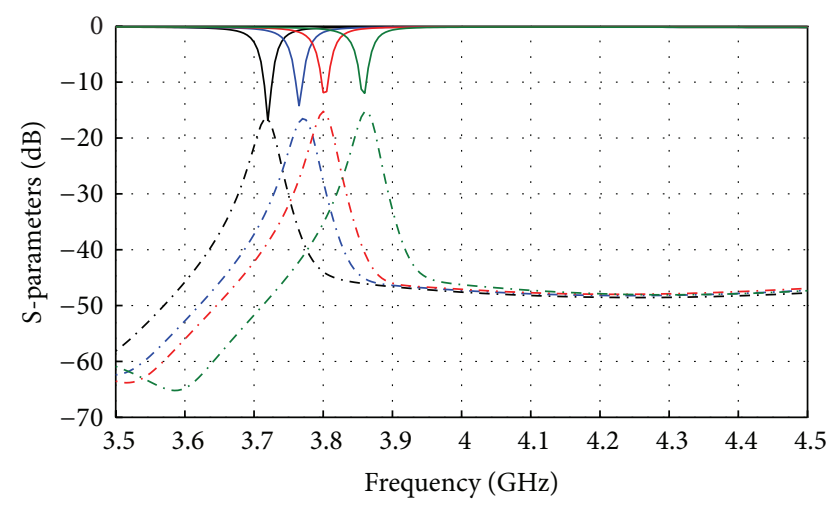

$$
\begin{array}{ll}
-S_{11}, V_{R}=0 \mathrm{~V} & -S_{11}, V_{R}=2 \mathrm{~V} \\
--S_{21}, V_{R}=0 \mathrm{~V} & -S_{21}, V_{R}=2 \mathrm{~V} \\
-S_{11}, V_{R}=1 \mathrm{~V} & -S_{11}, V_{R}=5 \mathrm{~V} \\
--S_{21}, V_{R}=1 \mathrm{~V} & --S_{21}, V_{R}=5 \mathrm{~V}
\end{array}
$$

FIGURE 14: Simulated $S$-parameters of the proposed pattern diversity tunable filter-antenna.

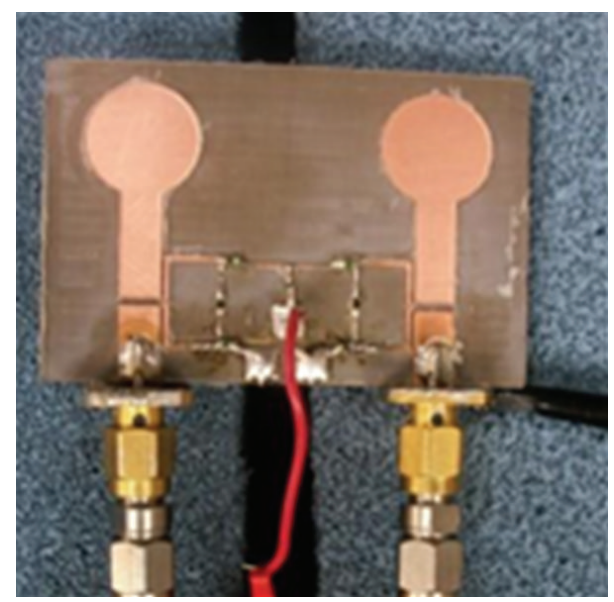

FIGURE 15: A fabricated prototype of the proposed pattern diversity tunable filter-antenna.

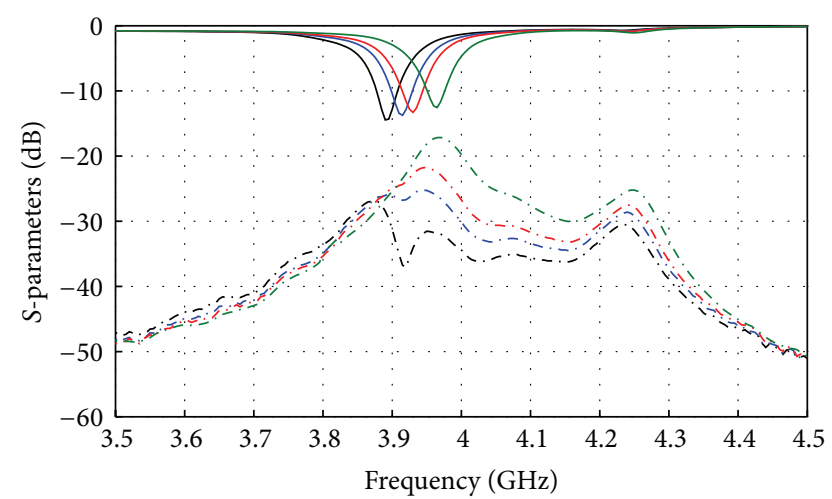

$$
\begin{array}{rr}
-S_{11}, V_{R}=0 \mathrm{~V} & -S_{11}, V_{R}=2 \mathrm{~V} \\
--S_{21}, V_{R}=0 \mathrm{~V} & -S_{21}, V_{R}=2 \mathrm{~V} \\
-S_{11}, V_{R}=1 \mathrm{~V} & -S_{11}, V_{R}=5 \mathrm{~V} \\
--S_{21}, V_{R}=1 \mathrm{~V} & --S_{21}, V_{R}=5 \mathrm{~V}
\end{array}
$$

FIGURE 16: Measured $S$-parameters of the proposed pattern diversity tunable filter-antenna.

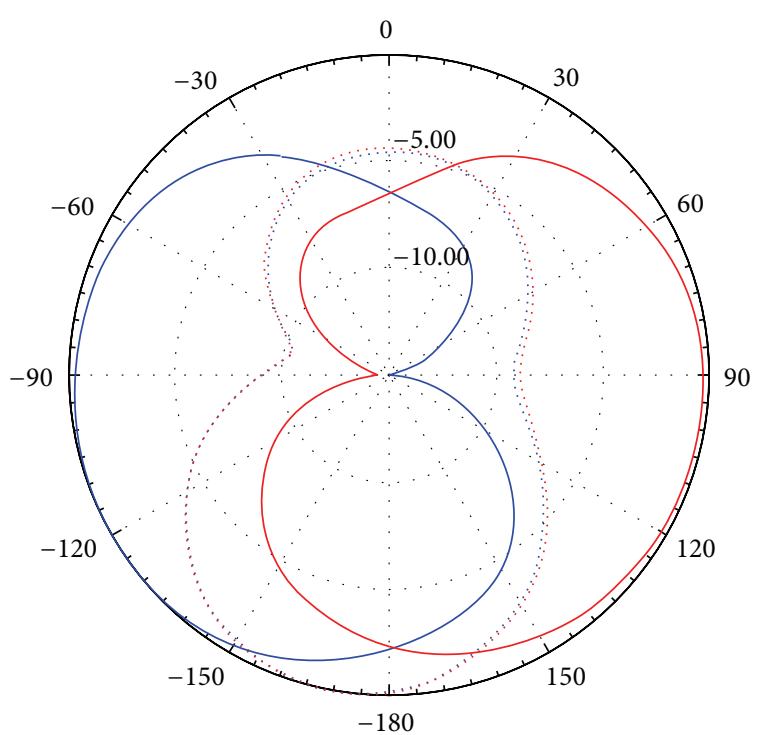

FIGURE 17: Normalized radiation pattern of the proposed pattern diversity tunable filter-antenna at $3.72 \mathrm{GHz}$ (X-Z/Y-Z plane: solid/dotted line).

received over the independent paths are then combined in such a way that the fading of the resultant signal is reduced. A wireless communication system equipped with diversity antennas leads to improved capacity and quality of the wireless channel. In this section, a pattern diversity antenna with a frequency tuning feature is presented. This will help combat multipath fading and cochannel interference, while sensing the frequency spectrum.

The proposed $1.6 \mathrm{~mm}$ thick Taconic TLY based pattern diversity tunable filter-antenna comprises two microstripline fed monopoles that are based on the same circular patch. Two symmetrically curved slots are etched in the ground plane. First, a circular slot with a radius of $8 \mathrm{~mm}$ is introduced below each patch, and then a $10 \times 18 \mathrm{~mm}^{2}$ rectangular slot is made at each of two corners. The etched curved slots direct the beams of the two monopoles in opposite azimuth directions, leading to pattern diversity. A $2 \times 20 \mathrm{~mm}^{2}$ rectangular slot placed halfway between the monopoles is used to increase the isolation and adjust the antenna's operating frequency range. Two varactor-tuned half-wavelength OLRbased bandpass filters are then integrated into the pattern diversity antenna to achieve frequency tuning. The detailed structure of the proposed pattern diversity tunable filterantenna is illustrated in Figure 13.

The computed $S$-parameters for each reverse voltage of the bridging varactor are shown in Figure 14. The simulations results reveal a narrowband frequency tuning and a better than $18 \mathrm{~dB}$ isolation. A fabricated prototype of the proposed design is depicted in Figure 15. The measured results are shown in Figure 16. A slight shift between the simulated and measured data is found due to SMV1405 modeling and milling issues. Figure 17 shows the normalized radiation patterns of the presented tunable filter-antenna at $3.72 \mathrm{GHz}$. It is seen that the patterns in the $\mathrm{X}-\mathrm{Z}$ plane are symmetric 
TABLE 1: Diversity parameters of the proposed tunable filterantenna.

\begin{tabular}{lcc}
\hline Frequency $(\mathrm{GHz})$ & $\rho_{e} \ll 0.5$ & MEG1/MEG2 $\approx 1$ \\
\hline 3.72 & $6214 \times 10^{-7}$ & 0.9952 \\
3.765 & $101 \times 10^{-4}$ & 1.0066 \\
3.8 & $7489 \times 10^{-7}$ & 0.9915 \\
3.86 & $3144 \times 10^{-6}$ & 0.9899 \\
\hline
\end{tabular}

and tend to cover complementary space region. With these patterns, the proposed design can provide pattern diversity over the operating frequencies. At $3.72 \mathrm{GHz}$, each monopole provides a peak gain of approximately $5 \mathrm{~dB}$.

The diversity performance of an antenna system, as reported in [11], can be assessed by calculating the envelope correlation coefficient $\left(\rho_{e}\right)$ and the mean effective gain (MEG). The correlation coefficient shows the influence of the different propagation paths on the RF signals reaching the antenna elements. In a diversity system, a good gain can be obtained when $\rho_{e}<0.5 . \rho_{e}$ relates to the mutual coupling between the antennas' ports as follows:

$$
\rho_{e}=\frac{\left|S_{11}^{*} S_{12}+S_{21}^{*} S_{22}\right|^{2}}{\left(1-\left|S_{11}\right|^{2}-\left|S_{12}\right|^{2}\right)\left(1-\left|S_{22}\right|^{2}-\left|S_{21}\right|^{2}\right)} .
$$

The mean effective gain is a parameter that includes antenna radiation power pattern, antenna efficiency, and the propagation effects. Equal power branches mean better condition for achieving high diversity gain. To guarantee that the signal strengths of the two monopoles are approximately equal, the ratio MEG1/MEG2 should be close to unity. For the proposed tunable filter-antenna, the conditions $\rho_{e}<0.5$ and MEG1/MEG2 $\approx 1$ are both met, per operating frequency, as given in Table 1 . As a result, a high diversity gain for the presented tunable filter-antenna is achieved:

$$
\text { MEG }=\frac{1}{2 \pi} \cdot \int_{0}^{2 \pi}\left[\frac{\Gamma \cdot G_{\theta}((\pi / 2), \varphi)+G_{\varphi}((\pi / 2), \varphi)}{1+\Gamma}\right] d \varphi .
$$

\section{Conclusion}

In this paper, tunable filter-antennas for overlay cognitive radio applications are presented. The exploitation of OLR to design a bandpass filter and its integration into a wideband antenna are discussed. The proposed approach is based on integrating varactor-tuned half-wavelength OLRbased bandpass filter(s) to electronically adjust the resonant frequency of the reported filter-antennas. Accordingly, a narrowband frequency-tunable microstrip antenna is firstly designed and tested. The same methodology is employed to design a pattern diversity tunable filter-antenna for overlay cognitive radio applications.

\section{Conflict of Interests}

The authors declare that there is no conflict of interests regarding the publication of this paper.

\section{Acknowledgments}

The authors gratefully acknowledge the support of the AUB Alumni Association Dubai \& Northern Emirates to the Electromagnetic and Radio Frequency Group (EMRF) at the American University of Beirut (AUB).

\section{References}

[1] E. Lourandakis, R. Weigel, H. Mextorf, and R. Knoechel, "Circuit agility," IEEE Microwave Magazine, vol. 13, no. 1, pp. 111121, 2012.

[2] A. Acampora, A. Collado, and A. Georgiadis, "Nonlinear analysis and optimization of a distributed voltage controlled oscillator for cognitive radio," in Proceedings of the IEEE International Microwave Workshop Series on RF Front-Ends for Software Defined and Cognitive Radio Solutions (IMWS '10), pp. 1-4, Aviero, Portugal, February 2010.

[3] B. Razavi, "Challenges in the design of cognitive radios," in Proceedings of the IEEE Custom Integrated Circuits Conference (CICC '09), pp. 391-398, San Jose, Calif, USA, September 2009.

[4] B. E. Carey-Smith, P. A. Warr, P. R. Rogers, M. A. Beach, and G. S. Hilton, "Flexible frequency discrimination subsystems for reconfigurable radio front ends," EURASIP Journal on Wireless Communications and Networking, vol. 2005, no. 3, pp. 354-363, 2005.

[5] T. Yücek and H. Arslan, "A survey of spectrum sensing algorithms for cognitive radio applications," IEEE Communications Surveys and Tutorials, vol. 11, no. 1, pp. 116-130, 2009.

[6] J. Perruisseau-Carrier, "Versatile reconfiguration of radiation patterns, frequency and polarization: a discussion on the potential of controllable reflectarrays for software-defined and cognitive radio systems," in Proceedings of the IEEE International Microwave Workshop Series on RF Front-Ends for Software Defined and Cognitive Radio Solutions (IMWS '10), pp. 1-4, Aviero, Portugal, February 2010.

[7] SMV1405-SMV1419, Hyperabrupt Junction Tuning Varactors from Skyworks Solutions.

[8] R. Marques, F. Martin, and M. Sorolla, Metamaterials with Negative Parameters: Theory, Design and Microwave Applications, John Wiley \& Sons, Hoboken, NJ, USA, 2008.

[9] Ansoft HFSS, Pittsburg, Pa 15219, USA.

[10] J.-S. Hong, "Theory and experiment of novel microstrip slow-wave open-loop resonator filters," IEEE Transactions on Microwave Theory and Techniques, vol. 45, no. 12, pp. 2358-2365, 1997.

[11] M. Al-Husseini, A. Ramadan, C. G. Christodoulou, K. Y. Kabalan, and A. El-Hajj, "A novel printed diversity antenna for WiMAX applications," in Proceedings of the IEEE International Symposium on Antennas and Propagation and USNC/URSI National Radio Science Meeting (APSURSI '11), pp. 2212-2214, Spokane, Wash, USA, July 2011. 

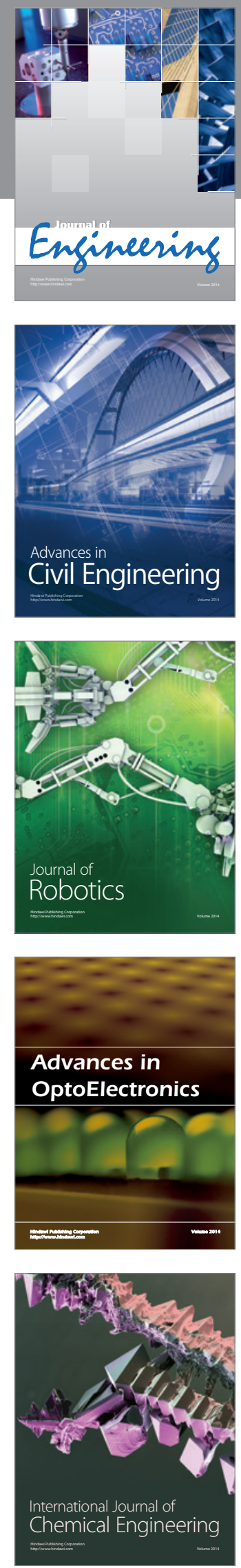

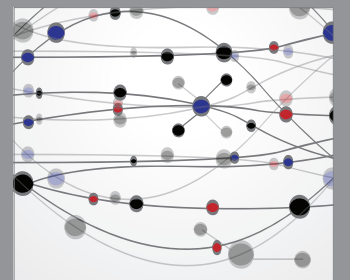

The Scientific World Journal
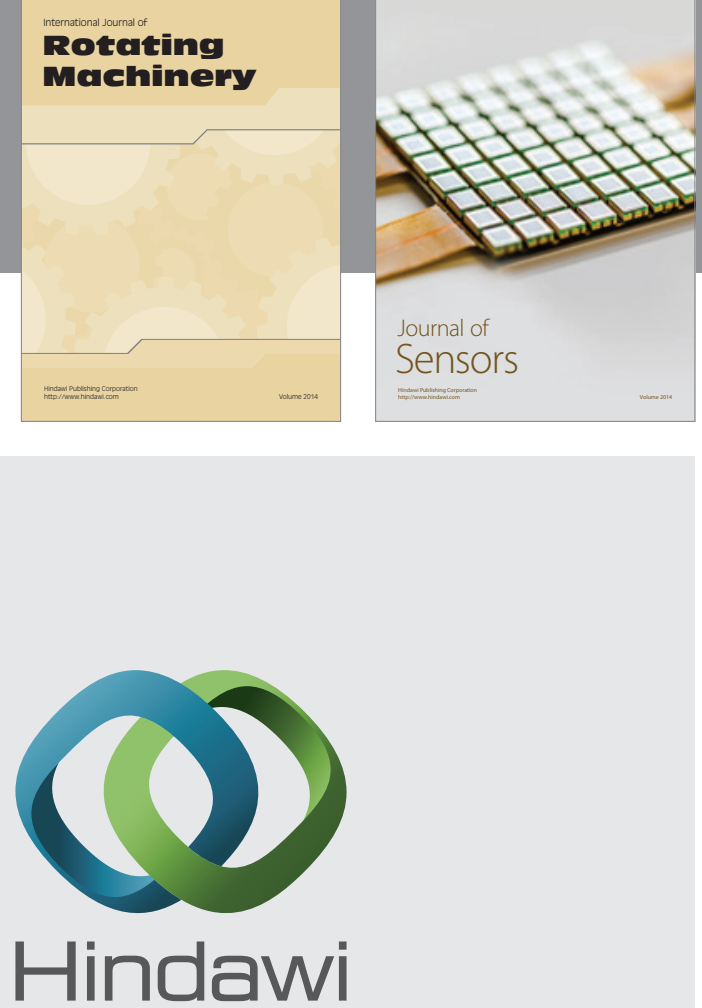

Submit your manuscripts at http://www.hindawi.com
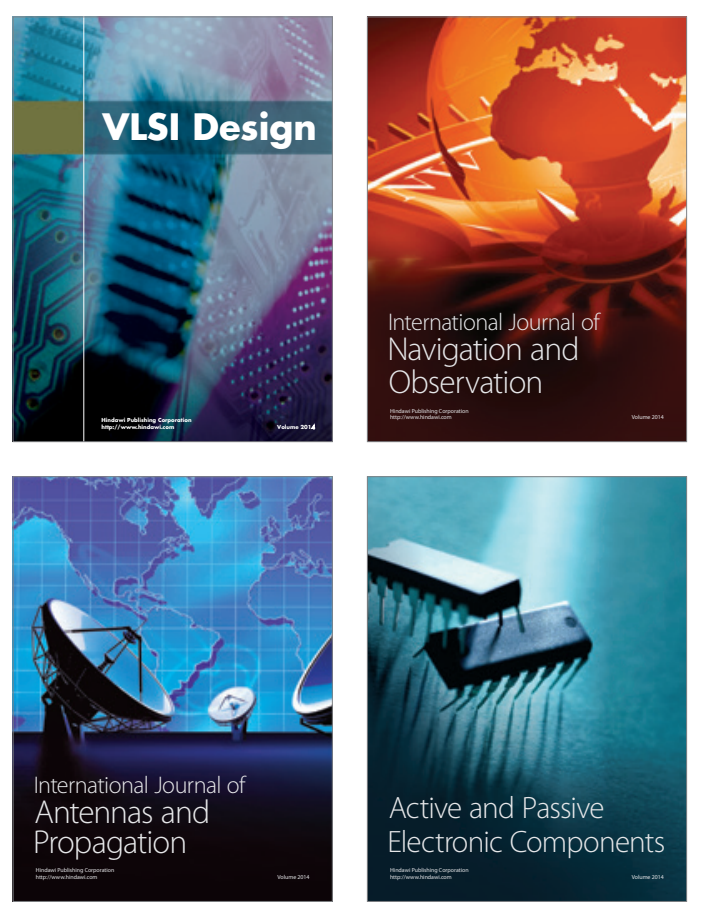
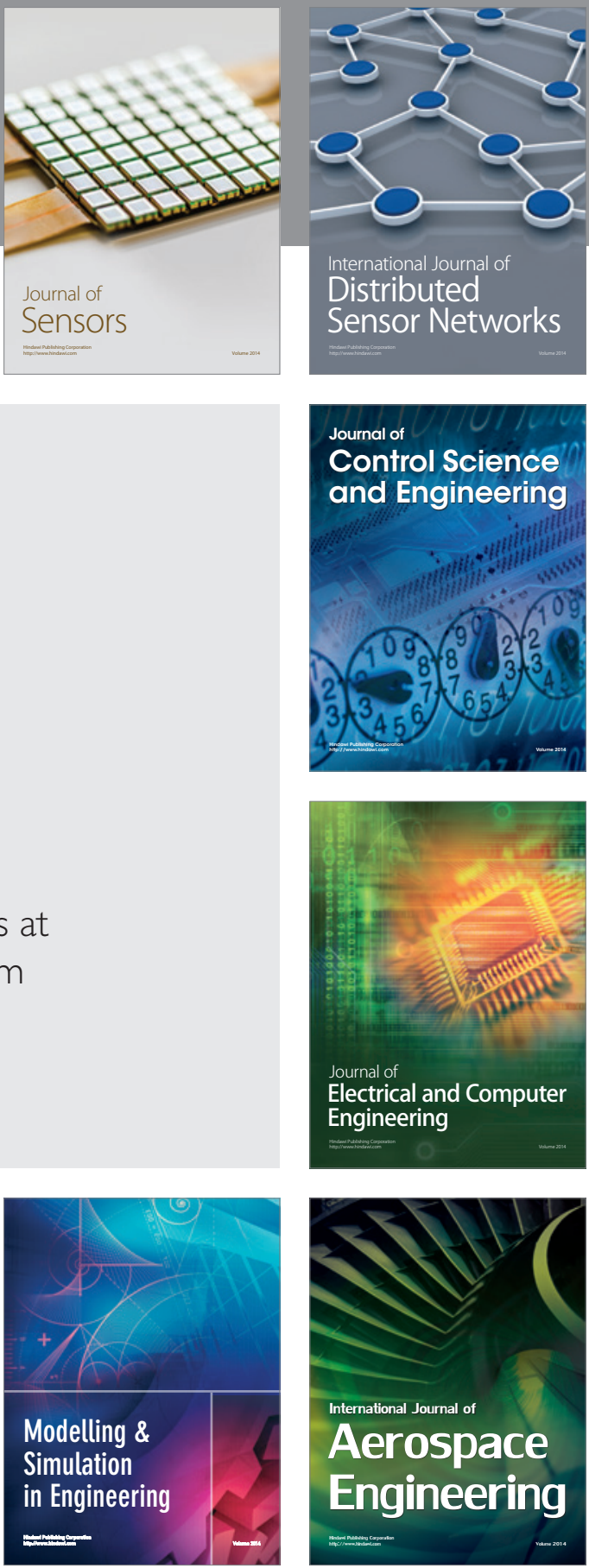

Journal of

Control Science

and Engineering
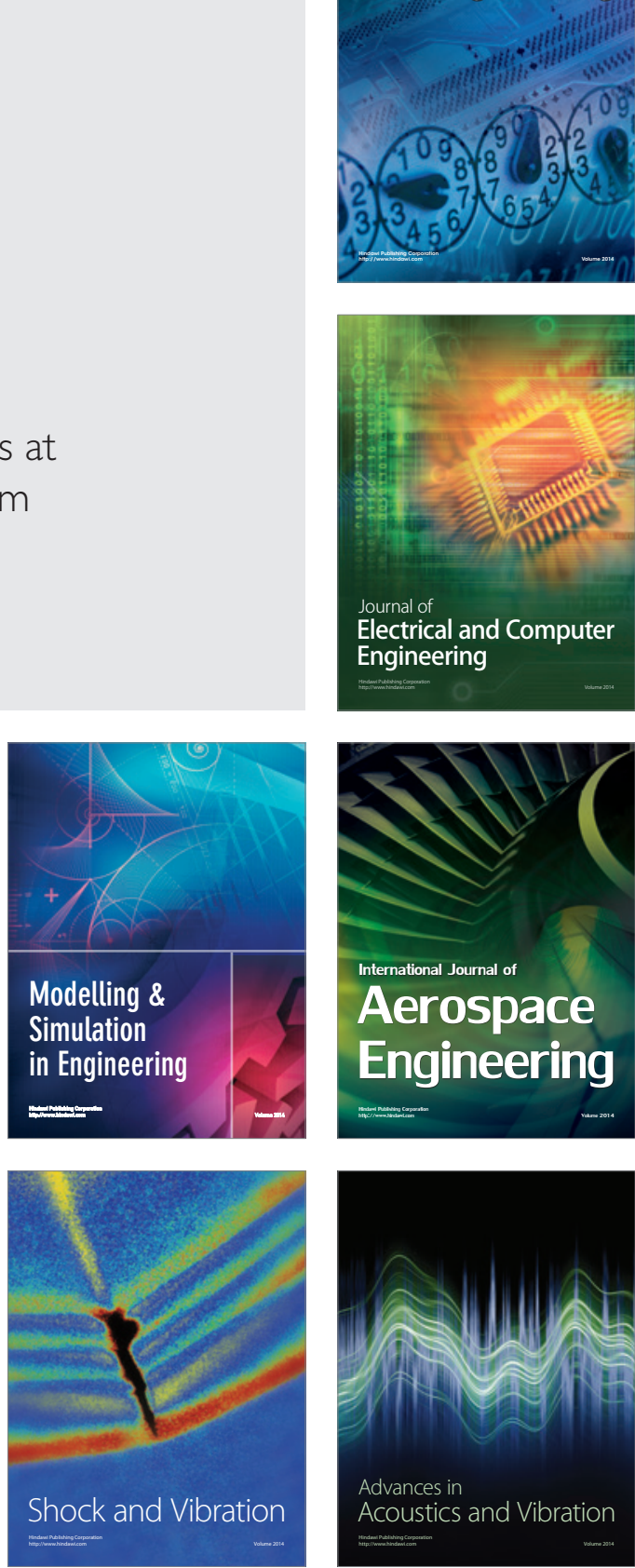